\title{
Erratum: Minimal distances for certain quantum product codes and tensor products of chain complexes [Phys. Rev. A 102, 062402 (2020)]
}

\author{
Weilei Zeng $\odot$ and Leonid P. Pryadko
}

(Received 24 June 2021; published 13 August 2021)

DOI: $10.1103 /$ PhysRevA.104.029902

Lemma 1 in our paper is not valid as originally stated. Here we give a formulation with a condition added. The condition is satisfied by all code families considered in the paper; hence it does not affect other results. We restate the lemma and its proof as follows.

Lemma 1 (Z-puncturing bound). Consider a stabilizer code $\mathcal{Q}=\operatorname{CSS}\left(H_{X}, H_{Z}\right)$ with the parameters $\left[\left[n, k,\left(d_{X}, d_{Z}\right)\right]\right]_{q}$ and a qudit index set $V=\{1,2, \ldots, n\}$. Given a partition into complementary sets $I \subset V$ and $J=V \backslash I$, suppose a logical generator matrix $L_{X}$ can be chosen so that none of its $k$ rows is supported both in $I$ and in $J$. Let $Q^{\prime}=\operatorname{CSS}\left(\left(H_{X}\right)_{I}, H_{Z}[I]\right)$ and $Q^{\prime \prime}=$ CSS $\left(\left(H_{X}\right)_{J}, H_{Z}[J]\right)$ be the codes whose $X$ generator matrices are shortened and $Z$ generator matrices punctured to $I$ and $J$, respectively. Then the $Z$ distances of the three codes satisfy the inequality $d_{Z} \geqslant \min \left(d_{Z}^{\prime}, d_{Z}^{\prime \prime}\right)$.

Proof. The case $k=0$ is trivial since it gives infinite $d_{Z}$. Assume $k>0$. The distance $d_{Z}$ of the code is the minimum weight in the set $Q_{Z}=\mathcal{C}_{H_{X}}^{\perp} \backslash \mathcal{C}_{H_{Z}}$ of all nontrivial $Z$-like codewords and their equivalent vectors. For any $c \in Q_{Z}$, the punctured vectors $c[I]$ and $c[J]$ are orthogonal to the rows of $\left(H_{X}\right)_{I}$ and $\left(H_{X}\right)_{J}$, respectively; the corresponding Pauli errors are undetectable. Further, since $L_{X} c^{T} \neq 0$, it is impossible that $c[I]$ is orthogonal to the rows of $\left(L_{X}\right)_{I}=L_{X}[I]$ and at the same time $c[J]$ is orthogonal to the rows of $\left(L_{X}\right)_{J}=L_{X}[J]$. Therefore, at most one of the vectors $c[I]$ and $c[J]$ can be trivial in the corresponding code.

Now consider the identity $\operatorname{wgt} c[I]+\operatorname{wgt} c[J]=\operatorname{wgt} c>0$. The punctured pieces $c[I]$ and $c[J]$ contribute to the distances $d_{Z}^{\prime}$ and $d_{Z}^{\prime \prime}$, respectively, only if the corresponding vectors are nontrivial. Let $d(c)$ equal infinity if $c$ is trivial in $\mathcal{Q}$ and wgt $c \geqslant 1$ otherwise; define similar functions $d^{\prime}(c)$ and $d^{\prime \prime}(c)$ for vectors corresponding to undetectable errors in $Q^{\prime}$ and $Q^{\prime \prime}$, respectively. Then $d_{Z}^{\prime} \leqslant \min _{c \in \mathcal{Q}_{Z}} d^{\prime}(c[I])$ and $d_{Z}^{\prime \prime} \leqslant \min _{c \in \mathcal{Q}_{Z}} d^{\prime \prime}(c[J])$. The stated result is obtained by minimizing the inequality $\min \left(d^{\prime}(c[I]), d^{\prime \prime}(c[J])\right) \leqslant d(c)$ over all $c \in Q_{Z}$.

The additional condition is needed to exclude the case where a codeword in $Q$ becomes trivial both in $Q^{\prime}$ and in $Q^{\prime \prime}$ after puncturing. For example, consider a qubit code $[[4,1,2]]_{2}$ with

$$
H_{X}=\left(\begin{array}{llll}
1 & 1 & 0 & 0 \\
0 & 0 & 1 & 1
\end{array}\right), \quad H_{Z}=\left(\begin{array}{llll}
1 & 1 & 1 & 1
\end{array}\right) .
$$

Then, with index sets $I=\{1,2\}$ and $J=\{3,4\}$, we get two trivial codes with no logical qubits and infinite distances, $d_{Z}^{\prime}=$ $d_{Z}^{\prime \prime}=\infty$, larger than $d_{Z}$. Indeed, it is easy to check by explicit enumeration that an $X$ codeword of the original code cannot be entirely supported on either $I$ or $J$. In comparison, with index sets $I=\{1,2,3\}$ and $J=\{4\}$, we may take $L_{X}=(0110)$ and $L_{Z}=\left(\begin{array}{llll}1 & 1 & 0 & 0\end{array}\right)$ to satisfy the conditions of the lemma; in this case we get $d_{Z}^{\prime}=2$ and a trivial code with $d_{Z}^{\prime \prime}=\infty$.

In our paper Lemma 1 is only used in Sec. IV $C$ to get lower bounds on the homological distance of a product of chain complexes. Here decomposition is only done along the block boundaries; the condition in Lemma 1 can be verified by explicitly constructing bases of the product chain and product cochain complexes and using the Künneth formula to make sure that no vectors are lost.

We thank Rui Chao for pointing out the defect of Lemma 1 in our paper. 